06

\title{
Излучение резонансной среды, возбуждаемое лазерным излучением с периодической фазовой модуляцией в режиме сильной связи поля и вещества
}

\author{
(C) P.М. Архипов ${ }^{1,2,3,{ }^{*}, \text { М.В. Архипов }}{ }^{1,2}$, B.С. Егоров $^{1}$, И.А. Чехонин ${ }^{1}$, М.А. Чехонин ${ }^{1}$, С.Н. Багаев ${ }^{4}$ \\ ${ }^{1}$ Санкт-Петербургский государственный университет, \\ 199034 Санкт-Петербург, Россия \\ ${ }^{2}$ Университет ИТМО, \\ 197101 Санкт-Петербург, Россия \\ ${ }^{3}$ Физико-технический институт им. А.Ф. Иофрфе, \\ 194021 Санкт-Петербург, Россия \\ ${ }^{4}$ Институт лазерной физики Сибирского отделения РАН, \\ 630090 Новосибирск, Россия \\ * e-mail: arkhipovrostislav@gmail.com
}

Поступила в редакцию 07.07.2019 г.

В окончательной редакции 07.07.2019 г.

Принята к публикации 12.08.2019 г.

\begin{abstract}
Теоретически изучено излучение двухуровневой резонансной среды, размещенной в резонаторе и возбуждаемой импульсом лазерного излучения с периодической модуляцией фазы. Анализ проведен на основе аналитического и численного решений системы уравнений Максвелла-Блоха в условиях, когда реализуется режим сильной связи поля и вещества. В этих условиях данная система аналогична поляритонному лазеру. Показана высокая эффективность возбуждения поляритонного лазера фазово-модулированным импульсом излучения по сравнению с импульсом без фазовой модуляции несущей частоты. Показано, что основной причиной эффективного возбуждения поляритонных мод среды является возникновение разностного комбинационного параметрического резонанса (difference combination parametric resonance). Полученные результаты открывают новые возможности в возбуждении излучения поляритонных лазеров лазерным излучением накачки малой мощности с частотной модуляцией.
\end{abstract}

Ключевые слова: поляритонный лазер, фазовая модуляция, сильная связь, вакуумное расщепление Раби.

DOI: $10.21883 /$ OS.2019.12.48694.180-19

\section{Введение}

Резонансное вещество, размещенное в оптическом резонаторе в условиях сильной связи поля и вещества, является объектом интенсивных теоретических и экспериментальных исследований в последние десятилетия (см. обзоры [1-3] и цитируемую в них литературу). В качестве таких объектов можно рассматривать, например, одиночные квантовые точки $[1,2]$, либо тонкие слои полупроводниковых структур, размещенные в микрорезонаторе [3]. Экситонные переходы в таких материалах могут активно взаимодействовать с модами резонатора, что приводит к различным интересным явлениям, таким как бозе-конденсация поляритонов [4,5], вакуумное расщепление Раби [1-3], формирование поляритонов связанных состояний фотонов и экситонов в резонатоpe $[6-16]$.

Изучение этих и других явлений, возникающих в подобной системе, имеет как фундаментальное, так и прикладное значение. Например, оно привело к работам по созданию нового класса источников когерентного оптического излучения - поляритонных лазеров при комнатной температуре [6-16]. Поляритонный лазер является компактным источником когерентного света без инверсии населенностей. Он существенно отличается от обычного лазера, для работы которого необходимо выполнение условия инверсии населенностей среды. Кроме того, для работы поляритонного лазера необходимо наличие режима сильной связи („strong coupling“) между полем и излучающими активными центрами в резонаторе. В связи с этим в последние десятилетия обсуждается возможное применение этих лазеров в различных оптических устройствах $[1-16]$.

Интересно отметить, что аналогичная система лазерный оптический резонатор с поглощающим веществом в режиме сильной связи поля и вещества в резонаторе - изучалась более 30 лет, до появления поляритонных лазеров, в методе внутрирезонаторной лазерной спектроскопии [17-24]. В данном методе ячейка с резонансным веществом, имеющим большие значения концентрации атомов и времен релаксации, помещалась в резонатор широкополосного лазера. В данной ситуации экспериментально наблюдается явление „конденсации спектра“, которое аналогично вакумному расщеплению Раби и заключается в формировании дублета интен- 
сивного излучения вблизи частоты перехода резонансно поглощающего вещества [17-24].

Для возбуждения поляритонной генерации обычно используются одиночные импульсы фемтосекундных лазеров большой мощности [1-9]. Однако данный механизм возбуждения является неэффективным в виду того, что только малая доля энергии накачки переходит в излучение на поляритонных частотах. Для повышения эффективности генерации в такой системе представляется интересным использование излучения с периодической модуляцией фазы или несущей частоты (FM). В этом случае частотная отстройка возбуждающего поля от частоты резонансного перехода среды периодически зависит от времени. В те моменты времени, когда мгновенная частота возбуждающего поля проходит через частоту резонанса среды, как мы увидим ниже, происходит быстрое возбуждение среды, которое происходит периодически с периодом модуляции частоты падающего поля. Это приводит к эффективному возбуждению генерации среды.

Отметим, что похожие явления, в основе которых лежит периодическая модуляция параметров системы, рассматривались ранее в оптике. Например, в работах $[25,26]$ показано, что периодическая модуляция атомной частоты перехода может приводить к генерации аттосекундных импульсов в атомной системе. В [27] изучается способ управления формой генерируемого гамма-излучения за счет периодической модуляции частоты переходов ядер. Периодическая модуляция размеров микрорезонатора с квантовыми точками может приводить к генерации запутанных состояний фотонов (entanglement generation) [28]. Задача взаимодействия FM-поля с атомными системами решалась аналитически в [29,30]. В [31] теоретически изучалось распространение и самовоздействие модулированного по частоте лазерного пучка в резонансной среде.

Учитывая указанный интерес к подобным системам, в настоящей работе теоретически изучается излучение системы резонансных двухуровневых атомов, помещенных в резонатор и возбуждаемых излучением с периодической модуляцией фазы или несущей частоты поля (FM-модуляция). Теоретический анализ проводился на основе аналитического и численного решения системы уравнений Максвелла-Блоха в условиях сильной связи поля и вещества. Аналитически изучаются свойства поляритонных частот и их времена жизни. Проанализирована эффективность возбуждения поляритонной генерации FM-полем по сравнению с фемтосекундным импульсом без FM-модуляции, но при той же энергии импульса.

\section{Теоретическая модель и свойства поляритонных мод}

В поляритонных лазерах резонансное вещество обычно помещено в микрорезонатор, имеющий размеры порядка длины световой волны, поэтому можно считать оправданным применение простой одномодовой модели системы. Для теоретического анализа рассматриваемой проблемы (система „поле + вещество“ в резонаторе) используется полуклассическая модель на основе системы уравнений Максвелла-Блоха для одной моды электромагнитного поля $E(t)$, поляризации $P(t)$ и разности заселенностей $N(t)$ двухуровневой среды, которая имеет вид [32-34]

$$
\begin{gathered}
\frac{d^{2}}{d t^{2}} E+\frac{1}{\tau} \frac{d E}{d t}+\omega_{r}^{2} E=-4 \pi \frac{d^{2}}{d t^{2}} P, \\
\frac{d^{2}}{d t^{2}} P+\frac{2}{T_{2}} \frac{d P}{d t}+\omega_{12}^{2} P=\frac{2 \omega_{12} d_{12}^{2}}{\hbar} N(t) E(t), \\
\frac{d N}{d t}+\frac{1}{T_{1}}\left(N-N_{0}\right)=-\frac{2}{\hbar \omega_{12}} E(t) \frac{d P}{d t} .
\end{gathered}
$$

Параметры модели: $\tau$ - время жизни излучения в резонаторе, $\omega_{r}$ - частота резонатора, $\omega_{12}$ - частота резонансного перехода вещества, $T_{1}$ - время релаксации разности заселенностей, $T_{2}$ - время релаксации поляризации, $d_{12}$ - дипольный момент перехода, $N_{0}$ разность заселенностей при отсутствии электромагнитного поля.

Уравнения (1), (2) аналогичны уравнениям двух связанных гармонических осцилляторов $-E(t)$ и $P(t)$. Система уравнений (1)-(3) легко допускает аналитический анализ при слабых полях, когда можно пренебречь изменением разности заселенностей $N \approx N_{0}$. Если искать решение в виде $E=E_{0} e^{i \Omega t}$ и $P=P_{0} e^{i(\Omega t+\varphi)}$, то нетрудно получить аналитическое выражение для собственных частот системы „поле + вещество“ $\Omega_{ \pm}$. Самой простой вид оно имеет при совпадении частоты резонатора с частотой перехода среды, $\omega_{r}=\omega_{12}$ :

$$
\begin{aligned}
\Omega_{ \pm} & =\omega_{12}+\frac{1}{2}\left(\frac{1}{2 \tau}+\frac{1}{T_{2}}\right) i \\
& \pm \frac{1}{2} \sqrt{4 \omega_{\text {coop }}^{2}-\left(\frac{1}{2 \tau}-\frac{1}{T_{2}}\right)^{2}} .
\end{aligned}
$$

Здесь $\omega_{\text {соор }}$ - кооперативная частота среды $[4,5,21,22]$,

$$
\omega_{\text {coop }} \equiv \sqrt{2 \pi \omega_{12} \frac{d_{12}^{2}}{\hbar} N_{0}} .
$$

Частоты $\Omega_{ \pm}$называются собственными частотами поляритонных мод системы (lower polariton branch, upper polariton branch).

Наличие режима сильной или слабой связи определяется соотношением между кооперативным временем среды $\left(T_{\text {соор }} \equiv \frac{2 \pi}{\omega_{\text {соор }}}\right)$ и временем релаксации поляризации среды $T_{2}$, а также временем жизни излучения в резонаторе $\tau$. Если кооперативное время среды $T_{\text {соор }}>T_{2}, \tau$, то энергия, передаваемая полем веществу, не может 
вернуться обратно к полю (режим слабой связи - weak coupling).

Наоборот, если кооперативное время среды меньше или сравнимо с временем релаксации поляризации вещества $T_{2}$ и временем затухания поля в резонаторе $\tau$, то возможен периодический нестационарный процесс обмена энергией между полем и средой. Поле многократно поглощается, а затем переизлучается средой (режим сильной связи - strong coupling). Такой обмен энергии наблюдается в оптически плотных средах. Частота этого процесса называется кооперативной частотой сре-

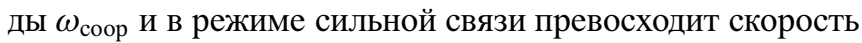
процессов релаксации (см. ниже).

Из (4) следует, что при

$$
\begin{aligned}
\omega_{\text {coop }} \gg \frac{1}{4}\left|\frac{1}{\tau}-\frac{2}{T_{2}}\right|, \\
\operatorname{Re}\left(\Omega_{ \pm}\right)=\omega_{12} \pm \omega_{\text {coop }}
\end{aligned}
$$

Это означает, что в режиме сильной связи у рассматриваемой продольной электромагнитной моды резонатора вместо одночастотного колебания появляется два нормальных колебания с близкими частотами, отстоящими от частоты перехода $\omega_{12}$ на величину кооперативной частоты. Отметим, что это расщепление физически связано с наличием периодического обмена энергией между полем и веществом. Таким образом, неравенство (6) определяет необходимое условие для существования сильной связи между полем и веществом в резонаторе $[4,5,21,22]$. Расщепление (7) по своей природе и есть вакуумное расщепление Раби, описанное многими авторами [1-5].

Важным следствием упомянутого аналитического расчета является тот факт, что время жизни электромагнитной моды в пустом резонаторе отличается от времени жизни моды в заполненном резонаторе в режиме сильной связи, когда скорость затухания поляритонных мод определяется выражением [34-36]

$$
\operatorname{Im}\left(\Omega_{ \pm}\right)=\frac{1}{4}\left(\frac{1}{\tau}+\frac{2}{T_{2}}\right) .
$$

Из (8) видно, что при $T_{2} \ll \tau$ скорость затухания поля поляритонной моды определяется временем $2 T_{2}$. Наоборот, когда $T_{2} \gg \tau$, скорость затухания моды определяется временем $4 \tau$ и оказывается в 2 раза больше, чем соответствующее время $2 \tau$ для моды электромагнитного поля в пустом резонаторе [34-36].

Факт увеличения времени жизни поляритонной моды до 2 раз остается справедливым и в сильных полях, когда нельзя пренебречь изменением разности заселенностей в уравнении (3) [34-36]. Это обстоятельство подтверждается также численным решением системы уравнений Максвелла-Блоха (1)-(3).

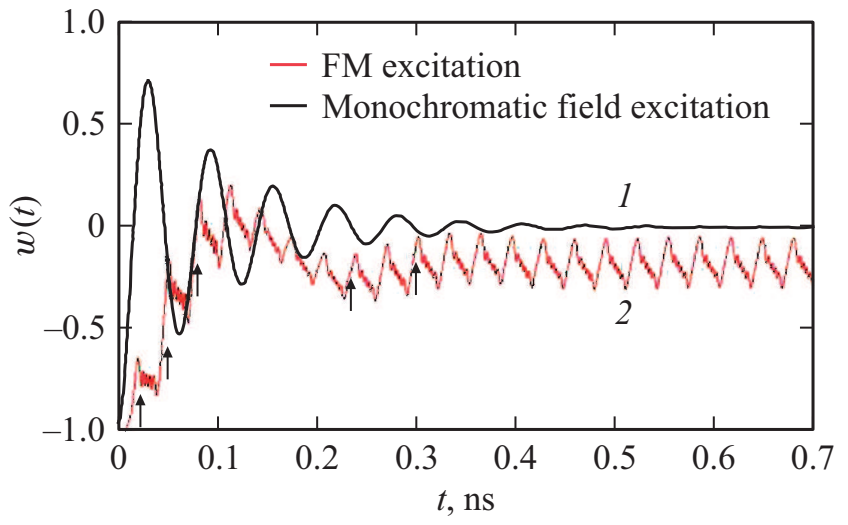

Рис. 1. Динамика разности заселенностей $w(t)$ при резонансной монохроматической накачке (черная линия, 1) и при нерезонансной FM-накачке (красная линия, 2).

\section{Возбуждение одиночного двухуровневого атома излучением с периодической модуляцией фазы}

Ниже рассматривается излучение ансамбля двухуровневых атомов в резонаторе, возбуждаемых излучением с периодической модуляцией фазы в режиме сильной связи поля и вещества. Интерес к данной проблеме связан с активным изучением поляритонных лазеров в последнее время, для накачки которых, как упоминалось выше, применяют одиночные фемтосекундные импульсы лазерного излучения. Как будет показано ниже, изученный в данном разделе способ является более эффективным. Для более глубокого понимания данного вопроса представляется интересным рассмотреть сначала возбуждение одиночного атома.

Поведение одиночного двухуровневого атома в поле накачки $E_{s}(t)$ описывается системой укороченных оптических уравнений Блоха для синфазной с полем $u(t)$, квадратурной $v(t)$ компонент поляризации и разности заселенностей $w(t)$ одиночного атома [32]:

$$
\begin{gathered}
\frac{d u}{d t}=-\Delta v-\frac{1}{T_{2}} u, \\
\frac{d v}{d t}=\Delta u-\frac{1}{T_{2}} v+\Omega_{\mathrm{R}} w, \\
\frac{d w}{d t}=-\frac{1}{T_{1}}(w+1)-\Omega_{\mathrm{R}} v .
\end{gathered}
$$

В этих уравнениях частотная расстройка $\Delta(t)$ определяется выражением (13), а $\Omega_{\mathrm{R}}=\frac{d_{12} E_{0}}{\hbar}$ частота Раби поля накачки.

Для двухуровневой системы, помещенной в резонансное монохроматическое лазерное поле с постоянной амплитудой, хорошо известно аналитическое решение Раби [32] системы (9)-(11). Согласно этому решению, на временах, меньших времен релаксации среды $T_{1}$ и $T_{2}$, 
атомная поляризация и разность заселенностей осциллируют с частотой Раби $\Omega_{\mathrm{R}}$ и стремятся к стационарному значению на временах, больших времен релаксации $T_{1}$ и $T_{2}$. При этом система насыщается [32]. Данное обстоятельство схематически изображено на рис. 1 .

Однако поведение двухуровневой системы, возбуждаемой излучением с периодической модуляцией фазы, оказывается более сложным и интересным. Данное FMполе имеет следующий вид:

$$
E s(t)=E_{0} \cos \left(\omega_{12} t+\Delta_{0} t+\Theta \sin \left(\Omega_{m} t\right)\right) .
$$

Мгновенная частотная расстройка такого поля от частоты атомного перехода $\omega_{12}$ имеет вид

$$
\Delta(t) \equiv \omega(t)-\omega_{12}=\Delta_{0}\left(1+m \cos \left(\Omega_{m} t\right)\right), m \equiv \frac{\Theta \Omega_{m}}{\Delta_{0}} .
$$

Здесь $\Omega_{m}$ - частота FM-модуляции, $m-$ индекс модуляции.

В этой задаче существенно, что поле накачки $E_{s}(t)(12)$ задано источником накачки и не зависит от поля переизлучения атома. Поле $E_{s}(t)$ имеет широкий линейчатый эквидистантный спектр с частотным интервалом между компонентами, равным $\Omega_{m}$. Расстояние между центральной частотой спектра поля и частотой атомного перехода $\omega_{12}$ определяется величиной расстройки $\Delta_{0}$.

Задача о возбуждении одиночного двухуровневого атома FM-полем накачки решалась аналитически в работах [29-30]. Однако полученные аналитические выражения являются громоздкими и трудными для анализа. Наиболее оптимальным является численный расчет системы уравнений (9)-(11) с частотной расстройкой (13). Такой пример приведен на рис. 1 (красная линия) и рис. 2 при следующих параметрах: $\Omega_{\mathrm{R}}=10^{11} \mathrm{rad} / \mathrm{s}, \Delta_{0}=10^{12} \mathrm{rad} / \mathrm{s}$, $\Omega_{m}=\Delta_{0} / 5=2 \cdot 10^{11} \mathrm{rad} / \mathrm{s}, m=0.99, T_{1}=T_{2}=0.1 \mathrm{~ns}$.

В те моменты времени, когда мгновенная расстройка приближается к 0, изменения $u(t), v(t)$ и разности заселенностей $w(t)$ происходят быстрым, ступенчатым (неадиабатическим) образом. Эти „скачки“ выделены стрелками на рис. 1 и 2.

При таких быстрых (неадиабатических) переходах в атоме происходит многократная необратимая передача энергии от поля $E s(t)$ к атомной системе (рис. 1 и 2). Данное обстоятельство может приводить к эффективному возбуждению ансамбля атомов в резонаторе и будет использовано в следующем разделе при анализе возбуждения ансамбля двухуровневых атомов в резонаторе.

\section{Излучение ансамбля двухуровневых атомов в резонаторе, возбуждаемых излучением с периодической модуляцией фазы}

В данном разделе проводится анализ возбуждения ансамбля двухуровневых атомов излучением с пе-
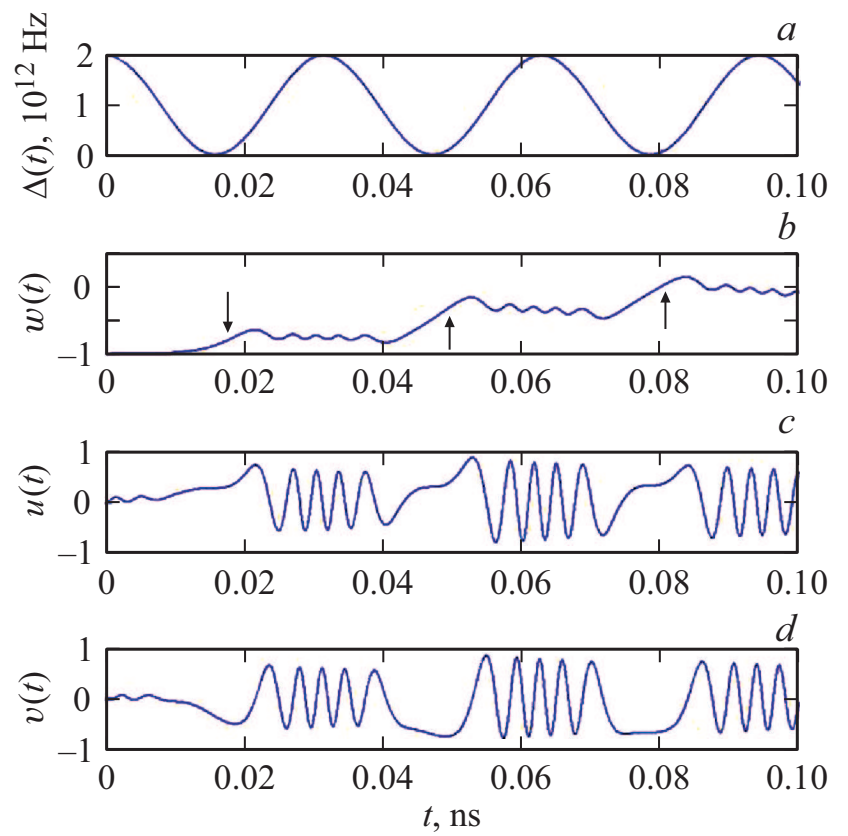

Рис. 2. Временная зависимость расстройки $\Delta(t)(a)$, разности заселенностей $w(t)(b)$ и компонент поляризации $u(t)(c)$ и $v(t)(d)$ при FМ-накачке.

риодической модуляцией фазы (12). Данный анализ проводится на основе решения системы уравнений Максвелла-Блоха (1)-(3). Для их анализа удобно перейти к медленно меняющимся амплитудам электрического поля генерации системы $A(t), B(t)$ и поляризации $u(t)$, $v(t)$, которые вводятся следующим образом:

$$
\begin{gathered}
E(t)=A(t) \cos (\omega t)+B(t) \sin (\omega t), \\
P(t)=d_{12} N_{0} u(t) \cos (\omega t)+d_{12} N_{0} v(t) \sin (\omega t) .
\end{gathered}
$$

Подставляя (14) и (15) в исходную систему уравнений (1)-(3) и переходя к медленно меняющимся амплитудам поля, поляризации и разности заселенностей, нетрудно получить укороченную систему уравнений Максвелла-Блоха для медленно меняющихся амплитуд поля и поляризации:

$$
\begin{gathered}
\frac{d A}{d t}=-\frac{1}{2 \tau} A(t)-2 \pi \omega_{12} N_{0} d_{12} v(t), \\
\frac{d B}{d t}=-\frac{1}{2 \tau} B(t)-2 \pi \omega_{12} N_{0} d_{12} u(t), \\
\frac{d u}{d t}=-\frac{1}{T_{2}} u(t)+\frac{d_{12} w(t)}{\hbar} \\
\times\left(E_{0} \sin \left(\Delta_{0} t+\Theta \sin \left(\Omega_{m} t\right)\right)-B(t)\right), \\
\frac{d v}{d t}=-\frac{1}{T_{2}} v(t)+\frac{d_{12} w(t)}{\hbar} \\
\times\left(E_{0} \cos \left(\Delta_{0} t+\Theta \sin \left(\Omega_{m} t\right)\right)+A(t)\right),
\end{gathered}
$$




$$
\begin{aligned}
\frac{d w}{d t}= & -\frac{1}{T_{1}}(w-1)-\frac{d_{12} A(t) v(t)}{\hbar}+\frac{d_{12} B(t) u(t)}{\hbar} \\
& -\frac{d_{12} E_{0} v(t)}{\hbar} \cos \left(\Delta_{0} t+\Theta \sin \left(\Omega_{m} t\right)\right) \\
& -\frac{d_{12} E_{0} u(t)}{\hbar} \sin \left(\Delta_{0} t+\Theta \sin \left(\Omega_{m} t\right)\right) .
\end{aligned}
$$

В данной задаче существенно то, что поле, действующее на каждый атом ансамбля, является самосогласованным и является суперпозицией поля внешней накачки $E s(t)$ и полей излучения всех окружающих его атомов. Предположим также, что параметры системы таковы, что выполнено условие сильной связи (6). Тогда в системе имеет место вакуумное расщепление Раби, и спектр излучения системы („поляритонные моды“) представляет собой дублет (7): $\operatorname{Re}\left(\Omega_{ \pm}\right)=\omega_{12} \pm \omega_{\text {соор. }}$.

Для эффективного возбуждения ,поляритонной генерации“ ансамбля атомов в резонаторе необходимо, чтобы две какие-либо из частот широкополосной FM-накачки $E s(t)(12)$ совпали с поляритонными модами системы $\operatorname{Re}\left(\Omega_{ \pm}\right)=\omega_{12} \pm \omega_{\text {соор }}$. Это условие выглядит следующим образом:

$$
\Omega_{m}=\frac{2 \omega_{\text {coop }}}{n}, n=1,2,3 \ldots
$$

Тогда, как и в случае возбуждения одиночного атома, рассмотренного выше (рис. 1 и 2), в те моменты времени, когда мгновенная частота $E s(t)$ приближается к $\omega_{12}$, изменения поляризации и разности заселенностей также будут происходить быстрым, ступенчатым (неадиабатическим) образом. Это приводит к эффективному возбуждению излучения системы. Динамика интенсивности генерации и спектр, полученные в результате численного решения системы уравнений (16)-(20), приведены на рис. $3, a$ и $3, b$ соответственно. Для численного расчета были выбраны следующие параметры: $T_{1}=T_{2}=1 \mathrm{~ns}, \quad d_{12}=6 \cdot 10^{-18} \mathrm{ESU}, \quad N_{0}=2 \cdot 10^{13} \mathrm{~cm}^{-3}$, $\omega_{\text {coop }}=1.1 \cdot 10^{11} \mathrm{rad} / \mathrm{s}, \quad \tau=5 \mathrm{~ns}, \quad E_{0}=30 \mathrm{ESU}, \quad \Omega_{m}=$ $=2 \omega_{\text {coop }}, m=0.94, \Delta_{0}=195 \omega_{\text {coop }}$.

На рис. 3, $a$ видно, что разность заселенностей $w(t)$ (красная линия) имеет характерные скачки во временной динамике (острые пики на рисунке). В эти моменты быстрого периодического изменения $w(t)$ происходит периодическое нарастание интенсивности генерации на временном промежутке $[0-1.5] \mathrm{ns}$. Затем в связи с большой интенсивностью $I(t)$ происходит изменение разности населенностей $w(t)$ и, как следствие, происходит нелинейное рассогласование частот поляритонных мод с частотами поля накачки $E_{s}(t)(12),(21)$. Поэтому на промежутке времени 1.5-2ns происходит спад интенсивности генерации.

Важно отметить, что, как видно на рис. 3, $a$, в процессе генерации среды отсутствует инверсия разности заселенностей $w(t)$.

Спектры генерации системы и поля накачки приведены на рис. $3, b$. На рис. $3, b$ видно, что спектральная

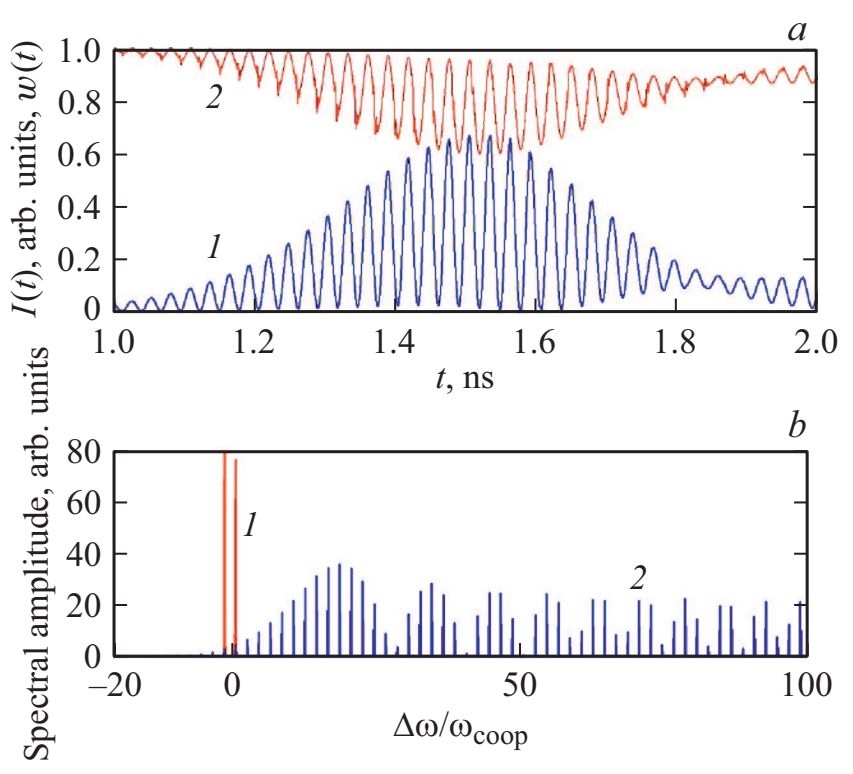

Рис. 3. (a) Динамика интенсивности излучения среды $I(t)=|E(t)|^{2}$ (в отн. ед.) (синяя линия, 1$)$ и разности заселенностей $w(t)$ (красная линия, 2). Верхняя линия $-w(t)$, нижняя линия - интенсивность поляритонной генерации. (b) Спектр излучения среды (красная линия, 1) и спектр накачки (синяя линия, 2).

интенсивность генерации ансамбля атомов может превышать интенсивность накачки в десятки раз. Данное обстоятельство может быть использовано для эффективного возбуждения поляритонного лазера (см. следующий раздел).

\section{Параметрический резонанс при FM-накачке системы „поле + вещество“}

В терминах теории колебаний описанный выше механизм возбуждения поляритонных мод системы „поле + вещество“ является параметрическим. Переменным параметром системы является частотная расстройка $\Delta(t)$ поля накачки. Чтобы убедиться в этом, сравним траектории вектора Блоха при FM-возбуждении одиночного атома и ансамбля двухуровневых атомов, помещенных в резонатор (поляритонный лазер).

Известно, что в приближении медленных огибающих амплитуд (SVEA) или в приближении вращающейся волны (RWA) оптические уравнения Блоха в отсутствие релаксации описываются уравнением прецессии вектора Блоха $\rho$ вокруг управляющего вектора $\Omega$ [32]:

$$
d \boldsymbol{\rho} / d t=[\boldsymbol{\Omega} \times \boldsymbol{\rho}],
$$

где $\boldsymbol{\rho}=[u, v, w] \quad-\quad$ вектор Блоха, $\boldsymbol{\Omega}=\left[-d_{12} E(t) / \hbar, 0, \Delta(t)\right]-$ управляющий $\quad$ вектор поля накачки. 
В режиме „адиабатического прохождения“ частоты поля через атомный резонанс происходит инверсия знака разности заселенностей $w$ [32]. В настоящей работе мы рассматриваем случай, когда режим „адиабатического прохождения“ нарушается и изменения $u(t), v(t)$ и разности заселенностей $w(t)$ происходят быстрым, ступенчатым (неадиабатическим) образом (рис. 1 и 2).

Пример траектории вектора Блоха при неадиабатическом FM-возбуждении одиночного атома на временном интервале [0-0.016] нс представлен на рис. 4. Параметры расчета: $\Omega_{\mathrm{R}}=1.7 \cdot 10^{11} \mathrm{rad} / \mathrm{s}, \Omega_{m}=2.4 \cdot 10^{11} \mathrm{rad} / \mathrm{s}$, $\Delta_{0}=200 \Omega_{m}, m=0.99, T_{1}=T_{2}=1 \mathrm{~ns}$. В промежутках между моментами неадиабатических скачков частотная расстройка поля $\Delta(t)$ велика, и атомная система излучает только спонтанно с временем релаксации $T_{1}$.

Возбуждение FM-полем ансамбля двухуровневых атомов, помещенных в резонатор, выглядит совершенно иначе. Хорошо известно, что если все атомы в одномодовом резонаторе локализованы на длине $l \ll \lambda$, то решение такой пространственно-однородной задачи сводится к нахождению траектории вектора Блоха - решению уравнения для нелинейного физического маятника (см., например, [37]).

При выполнении условия сильной связи (6) вектор Блоха совершает малые затухающие колебания с частотой $\omega_{\text {соор }}$ вблизи положения равновесия под действием самосогласованного поля излучения среды. Такой режим реализуется в случае слабого возбуждения среды, когда плотность фотонов в полости резонатора меньше плотности атомов $N_{0}$. Он соответствует рассмотренному выше режиму обратимого обмена энергией между полем и веществом с частотой $2 \omega_{\text {соор }}$.

На рис. 5 изображен фрагмент траектории вектора Блоха двухуровневой среды поляритонного лазера под действием самосогласованного поля излучения среды и FM-поля накачки с частотой модуляции $\Omega_{m}=2 \omega_{\text {coоp. }}$ На рисунке приведено решение уравнений Блоха (16)-(20) на временном промежутке одного цикла колебаний маятника [0.5645-0.5757] ns. Параметры расчета те же, что и на рис. 3 .

Неадиабатические кратковременные переходы в атомах ансамбля (рис. 5) приводят к периодическому изменению поляризации среды $u(t), v(t)$ и разности заселенностей $w(t)$ и параметрической раскачке нелинейного маятника, что и служит физической причиной увеличения амплитуды поля поляритонных мод среды.

В общем случае условие возбуждения параметрического резонанса имеет следующий вид: $\Omega_{m}=2 \omega_{\text {соор }} / n$, $n=1,2,3 \ldots$, что совпадает с условием (21).

Таким образом, стадия нарастания амплитуды поляритонных мод на рис. 3 соответствует параметрической раскачке колебаний маятника Блоха на временном промежутке 0-1.5ns, а стадия спада на промежутке 1.5-2ns - параметрическому демпфированию маятника.

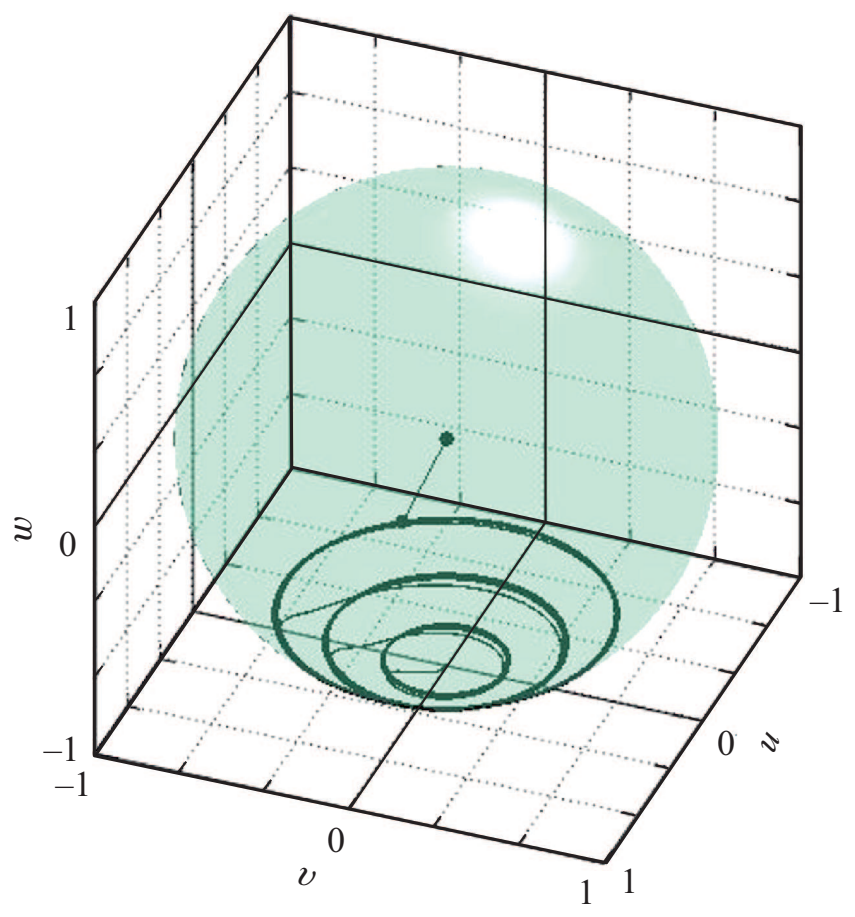

Рис. 4. Прецессия вектора Блоха одиночного атома под действием периодического FM-поля накачки.

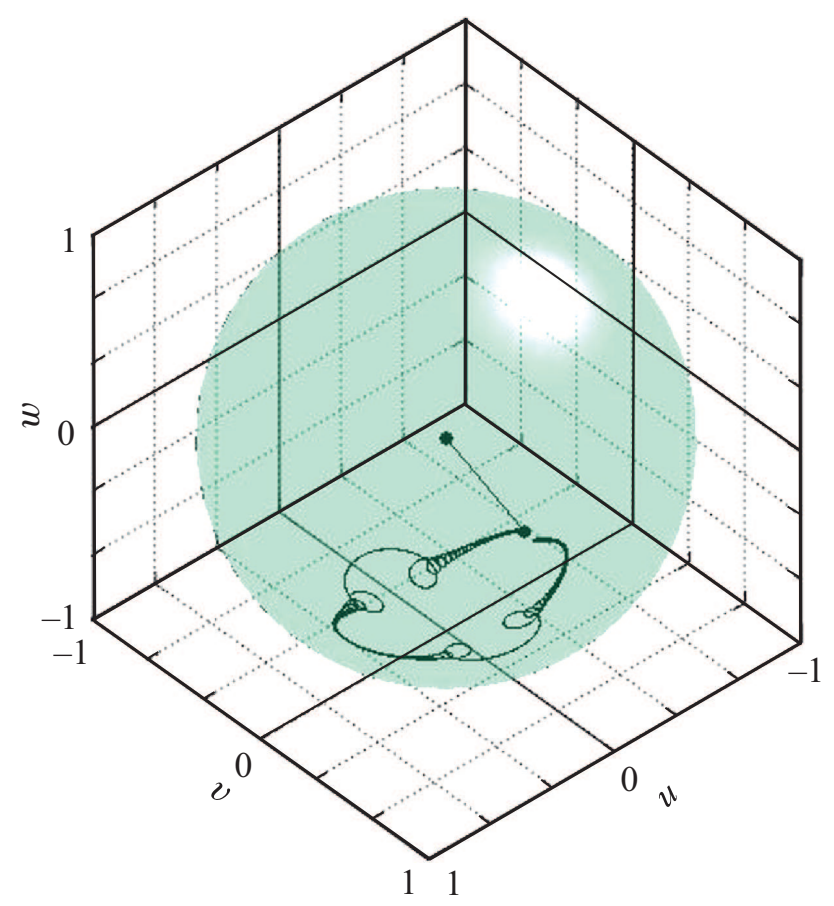

Рис. 5. Прецессия вектора Блоха под действием самосогласованного поля излучения ансамбля атомов и периодического FM-поля накачки. 


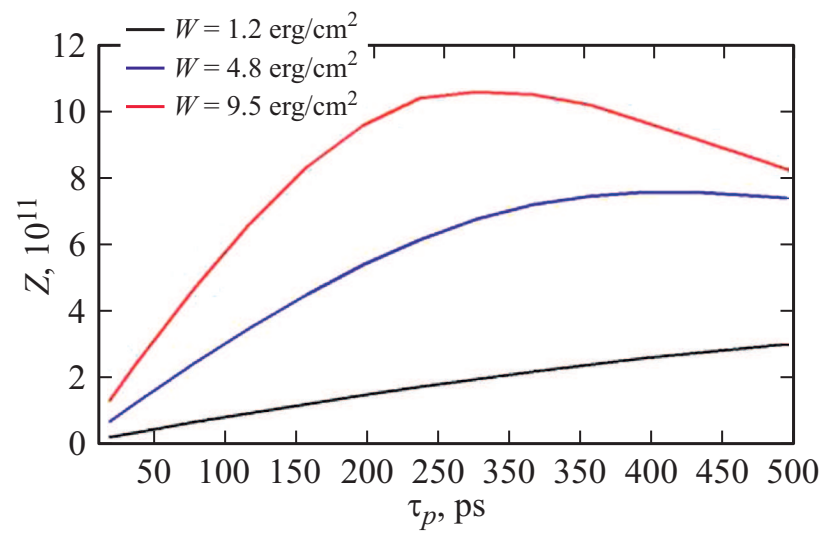

Рис. 6. Отношение $Z$ энергии генерации поляритонных мод в FM- и AM-режимах накачки в зависимости от длительности импульса FM-накачки. $\Delta \lambda_{0}=10 \mathrm{~nm}$.

В настоящее время описанный выше параметрический резонанс получил название разностного комбинационного параметрического резонанса (difference combination parametric resonance) [38,39]. По-видимому, впервые для системы „поле + вещество“ он был рассмотрен в [21] для случая АМ-модуляции поля нерезонансной накачки.

\section{Оценка эффективности FM-способа возбуждения поляритонной генерации среды}

Для оценки эффективности возбуждения поляритонного излучения резонансной среды нами исследовалось возбуждение системы короткими лазерными импульсами. Для этого система возбуждалась коротким гауссовым импульсом с заданной энергией $W$ без фазовой модуляции (АМ-импульс) и гауссовым импульсом с той же энергией $W$, но содержащим фазовую модуляцию, согласно формуле (12) (FМ-импульс).

Рисунок 6 иллюстрирует численно рассчитанное отношение $Z$ - энергии генерации на поляритонных модах при FM и АМ импульсных способах возбуждения в зависимости от длительности FM-импульса накачки $\tau_{p}$. Зависимости построены для трех значений энергии импульса $W$, при этом энергии импульсов с амплитудной и частотной модуляцией были одинаковыми. Длительность гауссового АМ-импульса накачки $\tau_{p}=200 \mathrm{fs}$, длительность гауссового FM-импульса варьировалась в пределах 0-500 ps. Отстройка центра спектра УКИ накачки $\Delta \lambda_{0}$ от частоты перехода была фиксирована и равна $10 \mathrm{~nm}$.

Из рис. 6 видно, что при импульсном FM-способе накачки эффективность $Z$ возбуждения поляритонного лазера возрастает в $10^{11}$ раз при равных энергиях импульсов накачки $W$.
Можно выделить два основных фактора столь значительного увеличения эффективности возбуждения поляритонных мод при FM-накачке. Первый - наличие разностного комбинационного параметрического резонанса (difference combination parametric resonance). Второй - неадиабатический квантовый скачок поляризации и разности заселенностей среды происходит при одновременном нелинейном взаимодействии всех мод лазера накачки с атомной системой независимо от ширины спектра лазерного излучения.

На основании проведенного исследования можно сделать вывод о том, что при FM-способе возбуждения можно получить быстрый рост значительных полей генерации среды, существенно снизив при этом мощность накачки.

Описанные выше неадиабатические квантовые скачки поляризации и разности заселенностей наблюдались экспериментально в [40] при накачке паров $\mathrm{Rb}$ одиночным импульсом лазера на титан-сапфире. Было показано, что введение частотной модуляции (чирпа) импульса накачки приводит к увеличению энергии импульса сверхизлучения паров рубидия на поляритонных модах среды.

При изменении знака чирпа импульса накачки происходил переход от эффекта „конденсации спектра“ эффекту „антиконденсации спектра“ вблизи частоты резонансных переходов рубидия $D_{1}$ и $D_{2}$. Спектр импульса сверхизлучения паров рубидия имел дублетную структуру, а поле импульса сверхизлучения представляло собой длительные биения поляритонных мод среды с частотой биений до $250 \mathrm{GHz}$.

\section{Заключение}

Таким образом, в работе на основе численного и аналитического решения системы уравнений Максвелла-Блоха изучено излучение резонансной среды, помещенной в резонатор, возбуждаемое лазерным полем с периодической модуляцией фазы в режиме сильной связи поля и вещества. Показано, что увеличение эффективности предложенного метода может достигать $10^{11}$ раз по сравнению со случаем возбуждения системы одиночным коротким импульсом без фазовой модуляции.

Показано, что основной причиной эффективного возбуждения поляритонных мод среды является возникновение разностного комбинационного параметрического резонанса (difference combination parametric resonance).

Данный FM-способ возбуждения может быть использован для эффективного возбуждения генерации поляритонных лазеров. Исследованный эффект открывает возможность использования лазерных диодов малой мощности для накачки одномодовых поляритонных лазеров.

Аналитически показано, что в режиме сильной связи поля и вещества время жизни излучения в резонаторе 
увеличивается в 2 раза по сравнению с временем жизни излучения в резонаторе. Данный, немного парадоксальный факт связан с взаимодействием двух связанных осцилляторов в резонаторе - поля и поляризации и периодического обмена энергии между ними в режиме сильной связи поля и вещества. Несмотря на то что данное явление описано в полуклассическом приближении, оно имеет аналог в квантовой электродинамике (эффект Парселла), связанный с увеличением времени спонтанного перехода атома, помещенного в резонатор, в режиме сильной связи атомного осциллятора и моды резонатора $[41]$.

\section{Финансирование работы}

Работа выполнена при поддержке гранта Российского научного фонда № 17-19-01097.

\section{Конфликт интересов}

Авторы заявляют, что у них нет конфликта интересов.

\section{Список литературы}

[1] Reitzenstein S. // IEEE J. Selected Topics in Quant. Electron. 2012. V. 18. N 6. P. 1733.

[2] Lodahl P., Mahmoodian S., Stobbe S., Mahmoodian S., Stobbe S. // Rev. of Mod. Phys. 2015. V. 87. N 2. P. 347.

[3] Schneider C., Glazov M.M., Korn T., Höfling S., Urbaszek B. // Nature Commun. 2018. V. 9. N 1. P. 2695.

[4] Kavokin A., Baumberg J., Malpuech G., Laussy $F$. Microcavities. Oxford University Press, Oxford, 2011.

[5] Sanvitto D., Timofeev V. Exciton Polaritons in Microcavities: New Frontiers V. 172. N. Y.: Springer Science \& Business Media, 2012.

[6] Brehier A., Parashkov R., Lauret J.S., Deleporte E. // Appl. Phys. Lett. 2006. V. 89. N 171110. P. 89.

[7] Tawara T., Gotoh H., Akasaka T. // NTT Technical Rev. 2005. V. 3. N 12. P. 27.

[8] Klimov V.I. // Los Alamos Science. 2003. N 28. P. 214-220.

[9] Saba M., Kundermann S., Ciuti C., Guillet T., Staehli J.L., Deveaud B. // Phys. Stat. Sol. B. 2003. V. 238. N 3. P. 432.

[10] Bajoni D., Wertz E., Senellart P., Miard A., Semenova E., Lemaitre A., Sagnes I., Bouchoule S., Bloch J. // Acta Phys. Polon. A. 2008. V. 114. P. 933.

[11] Kaspzak J., Richard M., André R., Le Si Dang // J. European Optical Society. 2008. V. 3. N 08023. P. 08023-1.

[12] Nomura M., Kumagai N., Iwamoto S., Otal Y., Arakawa Y. // Nature Phys. 2010. V. 6. P. 279.

[13] Kěna-Cohen S., Forrest S.R. // Nature Photon. 2010. V. 4. P. 371.

[14] Gibbs H.M., Khitrova G., Koch S.W. // Nature Photon. 2011. V. 5. N 5. P. 273.

[15] Kavokin A., Malpuech G., Gil B. Semiconductor Microcavities: Towards Polariton Lasers. Mater. Res. Soc. Internet J. Nitride Semicond., 2003.

[16] Lundt N., Marynski A., Cherotchenko E., Pant A., Fan X., Tongay S., Sek G., Kavokin A.V., Höfling S., Schneider C. // 2D Mater. 2016. V. 4. N 1. P. 015006.
[17] Гамалий В.Ф., Свириденков Э.А., Топтыгин Д.Д. // Квант. электрон. 1988. Т. 15. № 12. С. 2457; Gamaliu V.F., Sviridenkov E.A., Toptygin D.D. // Soviet J. Quant. Electron. 1988. V. 18. N 12. P. 1541.

[18] Баев В.М., Беликова Т.П., Варнавский О.П., Гамалий В.Ф., Коваленко С.А., Свириденков Э.А. // Письма в ЖЭТФ. 1985. Т. 42. № 10. С. 416; Baev V.M., Belikova T.P., Varnavskii O.P., Gamalii V.F., Kovalenko S.A., Sviridenkov E.A. // JETP Lett. 1985. V. 42. N 10. P. 514.

[19] Колеров А.Н. // Квант. электрон. 1988. Т. 15. № 3. С. 512; Kolerov A.N. // Soviet J. Quant. Electron. 1988. V. 18. N 3. P. 325.

[20] Baev V.M., Eschner J., Weiler A. // Appl. Phys. B. 1989. V. 49. P. 315.

[21] Васильев В.В., Егоров В.С., Чехонин И.А. // Опт. и спектр. 1985. T. 58. № 4. C. 944; Vasilev V.V., Egorov V.S., Chekhonin I.A. // Opt. Spectrosc. 1985. V. 58. N 4. P. 578.

[22] Васильев В.В., Егоров В.С., Федоров А.Н., Чехонин И.А. // Опт. и спектр. 1994. Т. 76. № 1. С. 146; Vasil'ev V.V., Egorov V.S., Fedorov A.N., Chekhonin I.A. // Opt. Spectrosc. 1994. V. 76. N 1. P. 134.

[23] Багаев С.Н., Егоров В.С., Морошкин П.В., Федоров А.Н., Чехонин И.А. // Опт. и спектр. 1999. Т. 86. № 6. С. 912; Bagaev S.N., Egorov V.S., Moroshkin P.V., Fedorov A.N., Chekhonin I.A. // Opt. Spectrosc. 1999. V. 86. N 6. P. 814.

[24] Bertseva E., Campargue A. // Optics Commun. 2004. V. 232. P. 251.

[25] Radeonychev Y.V., Polovinkin V.A., Kocharovskaya O. // Laser Phys. 2011. V. 21. N 7. P. 1243.

[26] Radeonychev Y.V., Polovinkin V.A., Kocharovskaya O. // Phys. Rev. Lett. 2010. V. 105. N 18. P. 183902.

[27] Vagizov F., Antonov V., Radeonychev Y.V., Shakhmuratov R.N., Kocharovskaya O. // Nature. 2014. V. 508 (7494). P. 80 .

[28] Blattmann R., Krenner H.J., Kohler S., Hänggi P. // Phys. Rev. A. 2014. V. 89(1). P. 012327.

[29] Alekseev A.V., Sushilov N.V. // Phys. Rev. A. 1992. V. 46. N 1. P. 351.

[30] Supplee J.M., Whittaker E.A., Andrew K. // JOSA B. 1998. V. 15. N 7. P. 1833.

[31] Пластун И.Л., Дербов В.Л. // Компьютерная оптика. 2009. T. 33. № 3. C. 233; Plastun I.L., Derbov V.L. // Computer Optics. 2009. V. 33. N 3. P. 233.

[32] Аллен Л., Эберли Джс. Оптический резонанс и двухуровневые атомы. М.: Мир, 1978; Allen L., Eberly J.H. Optical Resonance and Two-Level Atoms. N. Y.: Wiley, 1975.

[33] Ярив А. Квантовая электроника. М.: Сов. радио, 1980; Yariv A. Quant. Electron. John Wiley and Sons, Inc., 1975.

[34] Архипов Р.М., Егоров В.С., Чехонин И.А. // Научнотехн. вестник СПбГУ ИТМО. 2009. Т. 4. № 62. С. 53; Arkhipov R.M., Egorov V.S., Chekhonin I.A. // Nauch.-Tekh. Vestn. SPbGU ITMO. 2009. N. 4 (62). P. 53.

[35] Архипов Р.М., Егоров В.С., Чехонин И.А., Чехонин М.А., Багаев С.Н. // Труды VI Межд. конф. „Фундаментальные проблемы оптики-2010“. С. 112. СПб., Россия, 18-22 окт. 2010 г; Arkhipov R.M, Egorov V.S., Chekhonin I.A., Chekhonin M.A., Bagaev S.N. // Proc. VI Int. Conf. „Fundamental problems of optics-2010“. P. 112. St. Petersburg, Russia, Oct. 18-22. 2010. 
[36] Архипов М.В., Архипов Р.М., Егоров В.С., Чехонин И.А., Чехонин М.А., Багаев С.Н. // Изв. вузов. Приборостроение. 2012. № 7. C. 42; Arkhipov R.M., Arkhipov M.V., Egorov V.S., Chekhonin I.A., Chekhonin M.A., Bagaev S.N. // Izv. Vyssh. Uchebn. Zaved., Priborostroen. 2012. N 7. P. 42.

[37] Файн В.М., Ханин Я.И. Квантовая радиофизика. М.: Сов. радио, 1965; Fain V.M., Khanin Y.I. Quantum Radio Physics, V. 1: Photons and Nonlinear Media. Sov. Radio, M., 1972.

[38] Bagayev S.N., Arkhipov R.M., Arkhipov M.V., Egorov V.S., Chekhonin I.A., Chekhonin M.A. // J. Phys.: Conf. Ser. 2017. V. 917. P. 062028.

[39] Svidzinsky A.A., Yuan L, Scully M.O. // Phys. Rev. X. 2013. V. 3. P. 041001.

[40] Багаев С.Н., Преображенская А.А., Тимофеев Н.А., Пастор А.А., Мехов И.Б., Чехонин И.А., Сердобинцев П.Ю., Егоров В.С., Чехонин М.А., Машко А.М. // Опт. и спектр. 2018. Т. 125. № 5. С. 635; Bagaev S.N., Preobrazhenskaya A.A., Timofeev N.A., Pastor A.A., Mekhov I.B., Chekhonin I.A., Serdobintsev P.Yu., Egorov V.S., Chekhonin M.A., Mashko A.M. // Opt. Spectrosc. 2018. V. 125. N 5. P. 667.

[41] Ораевский А.Н. // УФН. 1994. Т. 164. № 4. С. 415; Oraevskii A.N. // Phys. Usp. 1994. V. 37. N 4. P. 393. 